\title{
Overexpression of heparan sulfate 6-O-sulfotransferase-2 enhances fibroblast growth factor-mediated chondrocyte growth and differentiation
}

\author{
WEI WANG ${ }^{1}$, XICHI JU ${ }^{2}$, ZHENGMING SUN ${ }^{3}$, WEIKUN HOU ${ }^{4}$, LIFANG YANG $^{1}$ and RUI ZHANG ${ }^{5}$ \\ ${ }^{1}$ Department of Prevention and Health Care, The Third Affiliated Hospital of the Medical College \\ of Xi'an Jiaotong University, Shaanxi Provincial People's Hospital, Xi'an, Shaanxi 710068; ${ }^{2}$ Department of Neurology, \\ The First Affiliated Hospital of Medical College of Xi'an Jiaotong University, Xi'an, Shaanxi 710061; \\ ${ }^{3}$ Department of Orthopaedics, The Third Affiliated Hospital of The Medical College of Xi'an Jiaotong University, Shaanxi \\ Provincial People's Hospital, Xi'an, Shaanxi 710068; ${ }^{4}$ Department of Joint Surgery and ${ }^{5}$ Laboratory of Orthopedics, \\ Xi'an Honghui Hospital, The Medical College of Xi'an Jiaotong University, Xi'an, Shaanxi 710054, P.R. China
}

Received January 1, 2015; Accepted June 19, 2015

DOI: $10.3892 /$ ijmm.2015.2272

\begin{abstract}
In our previous study, we reported that heparan sulfate 6-O-sulfotransferase-2 (HS6ST2) plays an important role in the cartilage of patients with osteoarthritis and Kashin-Beck disease and that it regulates aggrecan (Acan) metabolism and the viability of chondrocytes. However, its role in chondrocyte differentiation remains poorly understood. In the present study, we aimed to investigate the role of HS6ST2 in chondrocyte differentiation in vitro using mouse prechondrocytic cells. We found that the overexpression or silencing of HS6ST2 significantly enhanced or abrogated the effects of fibroblast growth factor (FGF)-2 on chondrocyte growth, respectively. We found that the overexpression of HS6ST2 significantly induced the expression of Acan as well as the amount of total proteoglycans in the prechondrocytic cells in the presence of FGF-2, whereas the silencing of HS6ST2 caused the opposite effect. Furthermore, the expresssion of FGF-2-induced sex-determining region Y-type high mobility group box protein 9 (SOX9), a major transcription factor for chondrocyte proliferation and differentiation, was also enhanced or blocked by HS6ST2 overexpression or HS6ST2 knockdown,
\end{abstract}

Correspondence to: Dr Xichi Ju, Department of Neurology, The First Affiliated Hospital of Medical College of Xi'an Jiaotong University, 277 Yanta West Road, Xi'an, Shaanxi 710061, P.R. China E-mail: juxichi@163.com

Abbreviations: HS6ST2, heparan sulfate 6-O-sulfotransferase-2; FGF, fibroblast growth factor; SOX9, sex-determining region Y-type high mobility group box protein 9; Acan, aggrecan; LV, lentiviral; FGFR, fibroblast growth factor receptor

Key words: heparan sulfate 6-O-sulfotransferase-2, fibroblast growth factor, chondrocyte respectively. Additionally, Wnt/ $\beta$-catenin signaling, which inhibited chondrocyte proliferation and differentiation, was suppressed by HS6ST2. Taken together, these data suggest that HS6ST2 plays an important role in regulating chondrocyte growth and differentiation by modulating FGF-2 signaling, thus indicating that it may be a potential and valuable molecular target for the treatment of skeletal dysplasias, such as dwarfism.

\section{Introduction}

Chondrocytes play an important role in skeletal development as they undergo proliferation and differentiation processes in the cartilage anlage and form a cartilaginous growth plate, thus contributing to endochondral ossification and bone growth (1-3). The dysregulation of chondrocyte growth and differentiation impairs endochondral ossification, leading to the development of severe skeletal dysplasias in human and animal models, such as dwarfism (4,5).

Heparan sulfate is a type of linear polysaccharide ubiquitously distributed in the extracellular matrix and cell surface that is capable of interacting with growth factors and receptors, and thus it affects numerous pathological and biological processes $(6,7)$. The functional chains of heparin sulfate are produced by Golgi-resident enzymes, such as the $\mathrm{O}$-sulfotransferases. The 6-O-sulfate content of heparan sulfate is catalyzed by heparan sulfate 6-O-sulfotransferases (HS6ST), which contribute to the transfer of sulphate to C-6 (an exocyclic carbon) of the glucosamine residue in heparan sulfate $(8,9)$. HS6ST2 is a member of the HSTST family that has been implicated in various cellular processes, such as organogenesis or tumorigenesis $(10,11)$. It has been suggested that the 6-O-sulfation of heparan sulfate contributes to the formation of a trimolecular complex which includes heparan sulfate, fibroblast growth factor (FGF) and its receptor (FGFR); of these, FGF is the most thoroughly investigated $(9,12,13)$.

It has been suggested that FGF plays a pivotal role in embryonic development, including limb development (14-16). Moreover, it has been reported previously that FGF-1 and FGF-2 
are expressed during chondrocyte differentiation $(17,18)$. The FGF receptors, including FGFR1a and FGFR2, have been shown to affect chondrocyte maturity and cartilage development in the pharyngeal endoderm (19). Murakami et al (20) demonstrated that FGF-2 promotes chondrocyte differentiation by upregulating the expression of sex-determining region Y-type high mobility group box protein 9 (SOX9) in primary chondrocytes and prechondrocytic cells. Previous studies have also reported that SOX9 is expressed in chondrocytes and prechondrocytic cells during embryonic development as a master transcriptional factor for chondrocyte differentiation, which is associated with regulating cartilage-specific proteins, such as aggrecan (Acan) $(21,22)$.

In recent years, studies have increasingly focused on the role of HS6ST2 in regulating cellular and pathological processes $(10,11)$. However, the role of HS6ST2 in chondrocyte growth and differentiation is not yet fully understood. In our previous study, we reported that HS6ST2 expression was significantly decreased in the cartilage of patients suffering from osteoarthritis and Kashin-Beck disease and was associated with cell proliferation and Acan metabolism in chondrocytes (23). These data suggest that HS6ST2 is involved in regulating chondrocyte growth. However, whether HS6ST2 is involved in regulating chondrocyte differentiation is an important matter which merits thorough investigation and clarification. Therefore, this study was carried out in order to determine the role of HS6ST2 in chondrocyte differentiation by modulating HS6ST2 expression. We demonstrate that HS6ST2 plays a critical role in regulating FGF-2-mediated chondrocyte growth and differentiation in vitro using prechondrocytic cells.

\section{Materials and methods}

Cell culture. Mouse prechondrocytic ATDC5 cells were purchased from the Cell Bank of Type Culture Collection of the Chinese Academy of Sciences (Shanghai, China). They were cultured in Dulbecco's modified Eagle's medium (DMEM; Life Technologies, Carlsbad, CA, USA) with $10 \%$ fetal bovine serum (FBS; Gibco, Rockville, MD, USA) containing $100 \mathrm{mg} / \mathrm{ml}$ streptomycin and $100 \mathrm{U} / \mathrm{ml}$ penicillin (Sigma, St. Louis, MO, USA) in a humidified atmosphere containing $5 \% \mathrm{CO}_{2}$ at $37^{\circ} \mathrm{C}$.

Cell transfection. Full-length mouse HS6ST2 cDNA was amplified and subcloned into the pcDNA3.1 mammalian expression vector. Null vectors (pcDNA3.1) without exogenous DNA fragments inserted were used as the controls. Lentiviral (LV)-shHS6ST2 and non-specific LV-shRNA were purchased from Santa Cruz Biotechnology, Inc. (Santa Cruz, CA, USA). Cell transfection was performed according to the manufacturer's instructions. To investigate gene overexpression, the plasmids $(1 \mu \mathrm{g})$ were diluted in $500 \mu \mathrm{l}$ of DMEM containing $5 \mu 1$ Lipofectamine (Invitrogen, Carlsbad, CA, USA) and added to the cells for incubation. For gene knockdown, LV-shRNA was transduced into the cells according to the manufacturer's instructions. Briefly, the cells were cultured in normal medium for $24 \mathrm{~h}$ before being cultured in medium containing Polybrene (Santa Cruz Biotechnology, Inc.). The cells were then infected with LV-shHS6ST2 or LV-shRNA $\left[0.5 \times 10^{5}\right.$ plaque-forming units (pfu)] overnight. The old medium was discarded and new medium without Polybrene was then added followed by incubation overnight. Stable clones expressing shRNA were selected with puromycin dihydrochloride (Santa Cruz Biotechnology, Inc.).

Alcian blue staining assay. The cells were transfected with pcDNA3.1-HS6ST2 or LV-shHS6ST2 in the presence of recombinant mouse FGF-2 (5 ng/ml; ProSpec, Rehovot, Israel) and incubated for 5 or 10 days. The cells were fixed with methanol at $-20^{\circ} \mathrm{C}$ for $40 \mathrm{~min}$, and this was followed by staining with $0.5 \%$ Alcian Blue 8 GX (Sigma, St. Louis, MO, USA) for $24 \mathrm{~h}$. After being washed with phosphate-buffered saline (PBS), the cells were lysed in $6 \mathrm{M}$ guanidine $\mathrm{HCl}$ for $6 \mathrm{~h}$. The absorbance at $630 \mathrm{~nm}$ was measured using a microtiter plate reader (Thermo Electron Corp., Vantaa, Finland).

Reverse transcription-quantitative polymerase chain reaction (RT- $q P C R)$. Total RNA was extracted using TRIzol reagent (Life Technologies) as per the manufacturer's instructions. cDNA was synthesized using Moloney Murine Leukemia Virus (M-MLV) reverse transcriptase (Clontech, Palo Alto, CA, USA) with $5 \mu \mathrm{g}$ of total RNA as the template. A mix containing the cDNA template, corresponding primers and SYBR-Green qPCR Master Mix (Thermo Fisher Scientific, Shanghai, China) was subjected to qPCR quantification (template denaturation step at $94^{\circ} \mathrm{C}$ for $4 \mathrm{~min}$; this was followed by 30 cycles of $20 \mathrm{sec}$ at $94^{\circ} \mathrm{C}, 30 \mathrm{sec}$ at $55^{\circ} \mathrm{C}$, and $20 \mathrm{sec}$ at $72^{\circ} \mathrm{C}$ ). Glyceraldehyde-3-phosphate dehydrogenase (GAPDH) was used as an internal control. Relative gene expression was quantified by normalization to GAPDH.

Western blot analysis. Proteins were extracted from the cells using a Total Protein Extraction kit (Applygen Technologies, Beijing, China), and the protein concentration was determined using the Bradford protein assay. A total of $25 \mu \mathrm{g}$ of protein was isolated by $12.5 \%$ sodium dodecyl sulfate-polyacrylamide gel electrophoresis (SDS-PAGE), and subsequently transferred onto nitrocellulose membranes (Bio-Rad, Hercules, CA, USA). The membranes were blocked with $2.5 \%$ non-fat milk for $1 \mathrm{~h}$ at $37^{\circ} \mathrm{C}$. Primary antibodies were diluted in blocking buffer and were then added followed by incubation overnight at $4^{\circ} \mathrm{C}$. After being washed with Tris-buffered saline containing 0.05\% Tween-20 (TBST), the membranes were incubated with goat anti-rabbit polyclonal horseradish peroxidase (HRP) conjugated secondary antibodies (dilution 1:2,000) (bs-0295G-HRP; Bioss, Beijing, China) for $1 \mathrm{~h}$ at room temperature. Subsequently, the membranes were washed again with TBST, and the immune-reactive protein bands on the membranes were detected using an enhanced chemiluminescence (ECL) detection system (Amersham, Little Chalfont, UK). The primary rabbit polyclonal anti-Acan antibodies (sc-25674; dilution 1:300) used in these experiments were purchased from Santa Cruz Biotechnology, Inc.; rabbit polyclonal anti-HS6ST2 antibodies (bs-17394R; dilution 1:200), rabbit polyclonal anti-SOX9 antibodies (bs-4177R; dilution 1:500) and rabbit polyclonal anti-GAPDH antibodies (bs-0459R; dilution 1:800) were purchased from Bioss. The protein gray value was measured using Image-Pro Plus 6.0 software (Media Cybernetics, Inc., Rockville, MD, USA). The fold changes were calculated by normalization to the control group. 

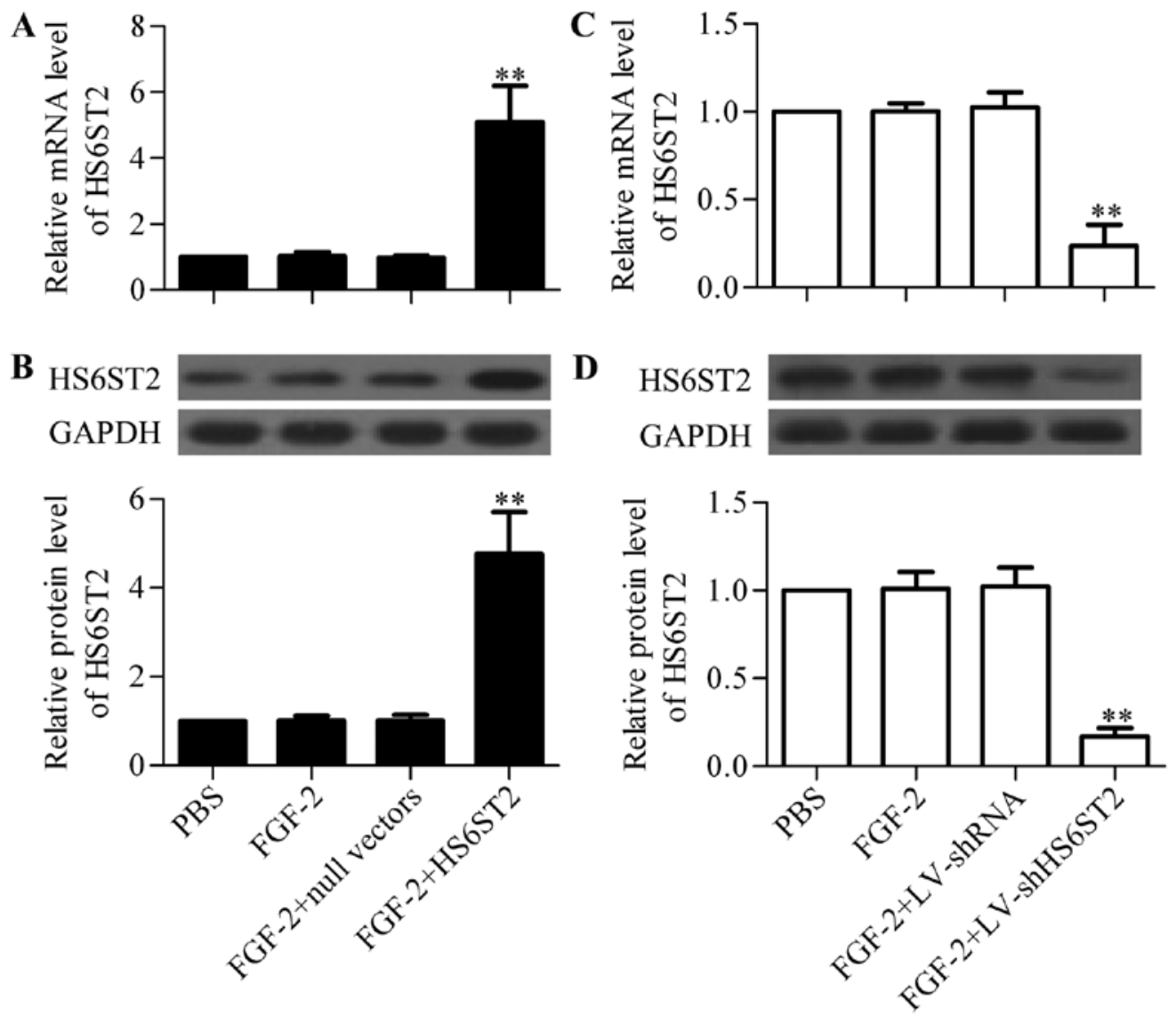

Figure 1. Expression of heparan sulfate 6-O-sulfotransferase-2 (HS6ST2) in the different cell groups. (A and C) RT-qPCR of the HS6ST2 mRNA expression levels in cells transfected with (A) pcDNA3.1-HS6ST2 or (C) lentiviral (LV)-shHS6ST2. Null vectors or LV-shRNA encoding non-specific shRNA were used as control groups, respectively. Fibroblast growth factor (FGF)-2 was administrated at a dose of $5 \mathrm{ng} / \mathrm{ml} . \mathrm{n}=3$; ${ }^{* *} \mathrm{p}<0.01$. (B and D) Western blot analysis of the HS6ST2 protein expression level in cells transfected with (B) pcDNA3.1-HS6ST2 or (D) LV-shHS6ST2. The protein levels were quantified using Image-Pro Plus 6.0 software and normalized to GAPDH. $n=3 ;{ }^{* *} \mathrm{p}<0.01$.

Cell growth and viability assay. Cell growth and viability were examined using the 3-(4,5-dimethylthiazol-2-yl)-2,5-diphenyltetrazolium bromide (MTT) assay. Cells stably expressing HS6ST2 or shHS6ST2 were seeded in 96-well plates at $1 \times 10^{4}$ cells/well in the absence of FGF-2 and incubated for 24, 48 and $72 \mathrm{~h}$. Subsequently, the old medium was discarded and fresh medium was added, with MTT solution $(5 \mathrm{mg} / \mathrm{ml}$ diluted in PBS) at $20 \mu \mathrm{l} /$ well. After being cultured for a further $4 \mathrm{~h}$, the formazan crystals which had formed were dissolved by the addition of dimethyl sulfoxide (150 $\mu \mathrm{l} /$ well; Sigma). The absorbance value was quantified at $490 \mathrm{~nm}$ using a microtiter plate reader (Thermo Electron Corp.).

Luciferase activity assays. Wnt signaling activity was measured by luciferase activity assay, which was indicated by the activity of the T-cell factor (TCF)-responsive reporter. The cells were co-transfected with pcDNA3.1-HS6ST2 or LV-shHS6ST2 and TCF-responsive reporter, TOPFlash Firefly luciferase reporter vector (Addgene, Cambridge, MA, USA) and Renilla luciferase vectors phRL-TK (Promega, Madison, WI, USA). Following transfection for $24 \mathrm{~h}$, the cells were harvested and lysed; the luciferase activities were quantified using the dual-luciferase reporter assay kit (Promega).

Data analysis. Data are reported as the means \pm standard deviation (SD). Statistical analysis was performed using SPSS version 5 (SPSS Inc., Chicago, IL, USA). One-way analyses of variance (ANOVA), followed by Bonferroni post-hoc tests, were employed to evaluate the statistical differences. Differences were considered statistically significant at p-value $<0.05$.

\section{Results}

HS6ST2 accelerates the FGF-2-mediated growth of chondrocytes. In order to investigate the role of HS6ST2 in chondrocytes, we transfected prechondrocytic ATDC5 cells with pcDNA3.1-HS6ST2 or LV-shHS6ST2, thus inducing the stable overexpression of HS6ST2 (Fig. 1A and B) or the silencing of HS6ST2 expression (Fig. 1C and D). We then investigated the effects of HS6ST2 overexpression on the growth of prechondrocytic ATDC5 cells. The results of MTT assay indicated that FGF-2 markedly increased the growth of the cells, and this growth was further enhanced by the overexpression of HS6ST2 (Fig. 2A). By contrast, the knockdown of HS6ST2 significantly inhibited the FGF-2-mediated growth of chondrocytes (Fig. 2B). Taken together, these results indicate that HS6ST2 plays an important role in the FGF-2-mediated growth of chondrocytes.

Overexpression of HS6ST2 enhances FGF-2-mediated chondrocyte differentiation. To examine the role of HS6ST2 in 

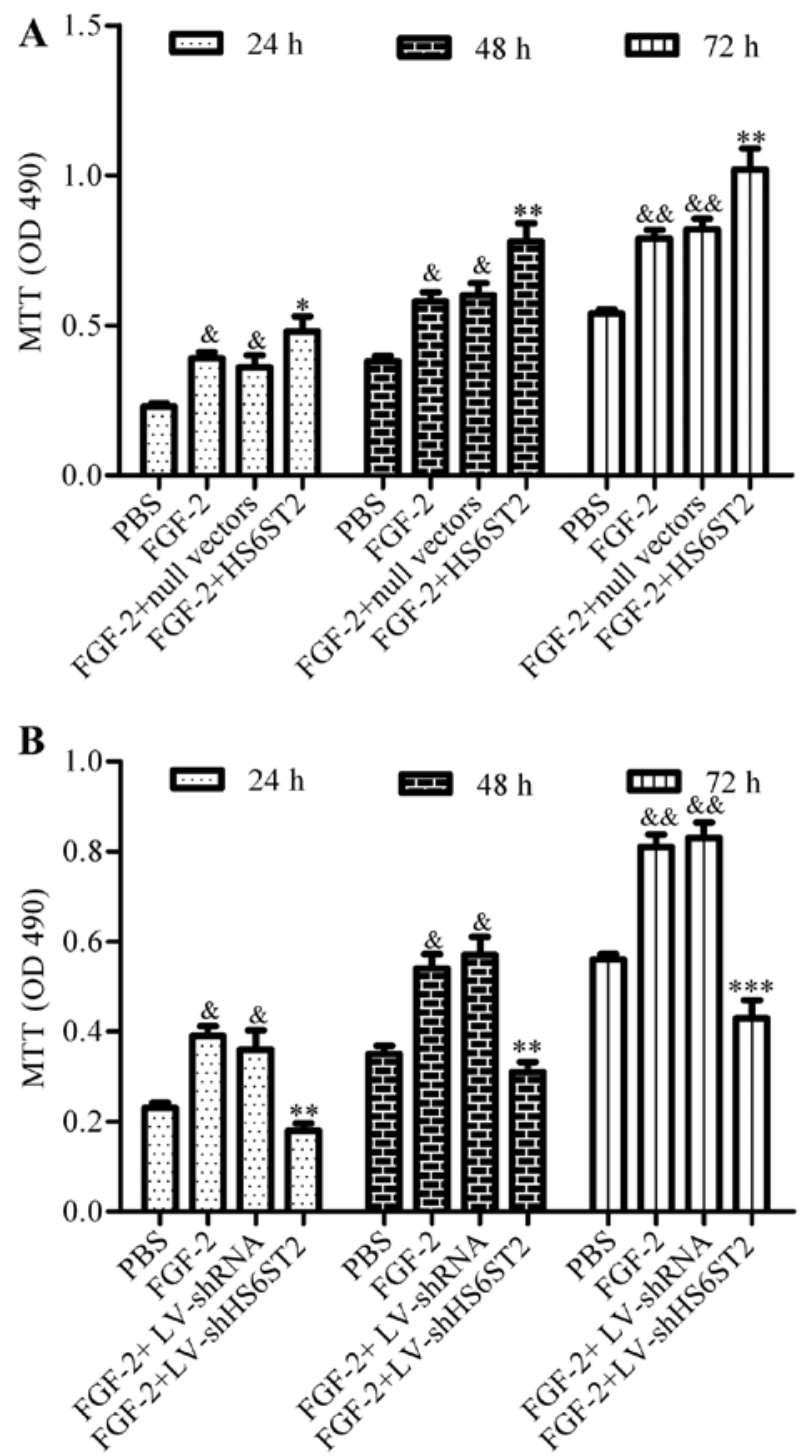

Figure 2. Role of heparan sulfate 6-O-sulfotransferase-2 (HS6ST2) in the fibroblast growth factor (FGF)-2-mediated growth of chondrocytes. (A) MTT assay of the effects of HS6ST2 overexpression on FGF-2-mediated cell growth. Cells were transfected with HS6ST2 overexpression vectors in the presence of FGF-2 $(5 \mathrm{ng} / \mathrm{ml})$ for 24,48 and $72 \mathrm{~h}$. Null vectors were used as control plasmids. $n=3 ;{ }^{\&} \mathrm{p}<0.05$ and ${ }^{\& \&} \mathrm{p}<0.01$ vs. PBS group; $\mathrm{p}<0.05$ and * $\mathrm{p}<0.01$ vs. FGF-2 or FGF-2 + null vectors. (B) MTT assay of the effects of HS6ST2 silencing on FGF-2-mediated cell growth. Cells were infected with lentiviral (LV)-shHS6ST2 in the presence of FGF-2 $(5 \mathrm{ng} / \mathrm{ml})$ for 24,48 or $72 \mathrm{~h}$. LV-shRNA encoding non-specific shRNA was used as a control. $\mathrm{n}=3$; ${ }^{*} \mathrm{p}<0.05$ and ${ }^{\& \&} \mathrm{p}<0.01$ vs. PBS group; ${ }^{* *} \mathrm{p}<0.01$ and ${ }^{* * *} \mathrm{p}<0.001$ vs. FGF-2 or FGF-2 + LV-shRNA group.

chondrocyte differentiation, we examined the expression of the chondrocyte differentiation-associated gene, Acan. The results from RT-qPCR demonstrated that the mRNA expression level of Acan was significantly increased by treatment with FGF-2, which was applied during ATDC5 differentiation for 5 or 10 days (Fig.3A). The overexpression of HS6ST2 further induced the mRNA expression levels of Acan in the presence of FGF-2 (Fig. 3A). Western blot analysis of the protein expression levels further confirmed the augmentary effect of HS6ST2 overexpression on the expression of Acan in the presence of FGF-2 (Fig. 3B). Moreover, HS6ST2 overex-
A
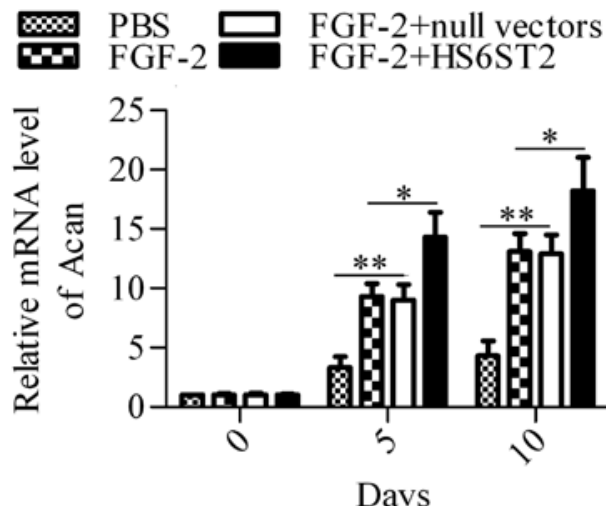

B
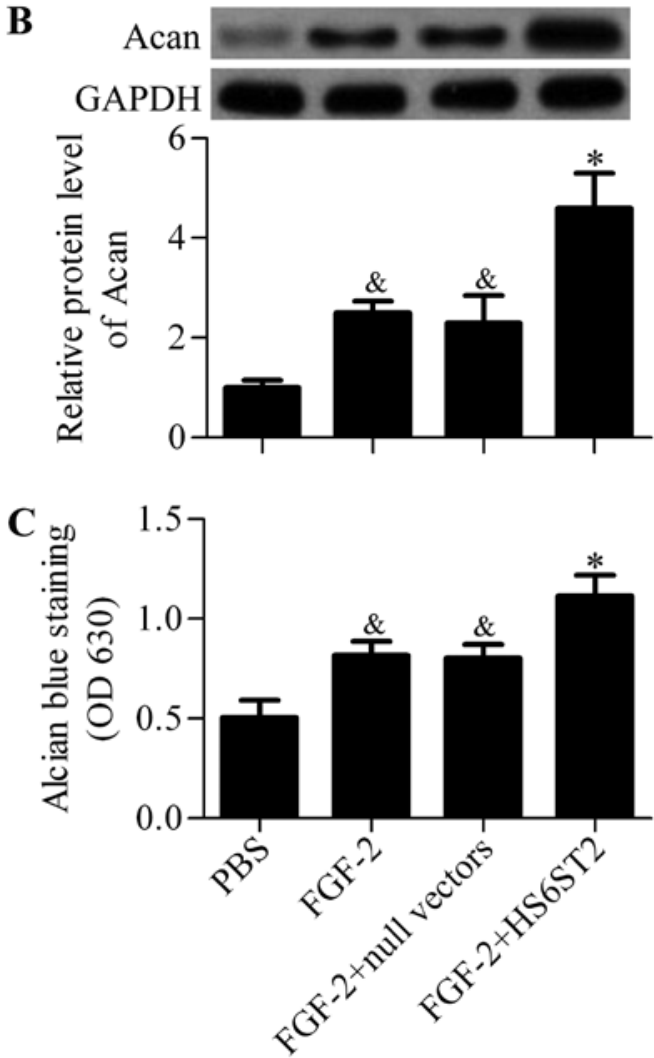

Figure 3. Effect of heparan sulfate 6-O-sulfotransferase-2 (HS6ST2) overexpression on fibroblast growth factor (FGF)-2-mediated chondrocyte differentiation. (A) RT-qPCR of the aggrecan (Acan) mRNA expression levels in the different cell groups. Cells were transfected with HS6ST2 overexpression vectors in the presence of FGF-2 ( $5 \mathrm{ng} / \mathrm{ml})$ for 5 or 10 days. $\mathrm{n}=3$; ${ }^{*} \mathrm{p}<0.05$ and ${ }^{* *} \mathrm{p}<0.01$ compared to day 0. (B) Western blot analysis of Acan protein expression on day 10.Protein levels were quantified using Image-Pro Plus 6.0 software and normalized to GAPDH. $n=3 ;{ }^{\&} \mathrm{p}<0.05$ vs. PBS group; *p $<0.05$ vs. FGF-2 or FGF-2 + null vectors. (C) Amount of proteoglycans detected by Alcian blue staining in the cell lysates. $n=3 ;{ }^{\&} p<0.05$ vs. PBS group; " $\mathrm{p}<0.05$ vs. FGF-2 or FGF-2 + null vectors.

pression significantly increased the amount of proteoglycans in the extracellular matrix (Fig. 3C). These findings indicate that the overexpression of HS6ST2 enhances chondrocyte differentiation induced by FGF-2.

Knockdown of HS6ST2 reduces FGF-2-mediated chondrocyte differentiation. To further investigate the effects of HS6ST2 on chondrocyte differentiation, we transfected the ATDC5 cells with LV-shHS6ST2, which silenced the expression of HS6ST2. 


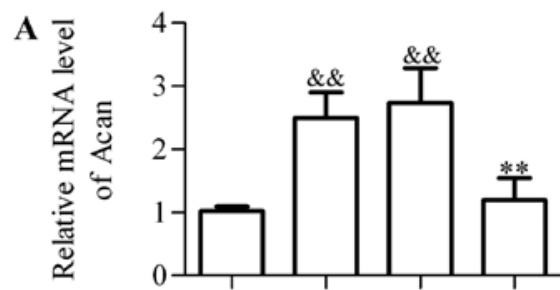

B
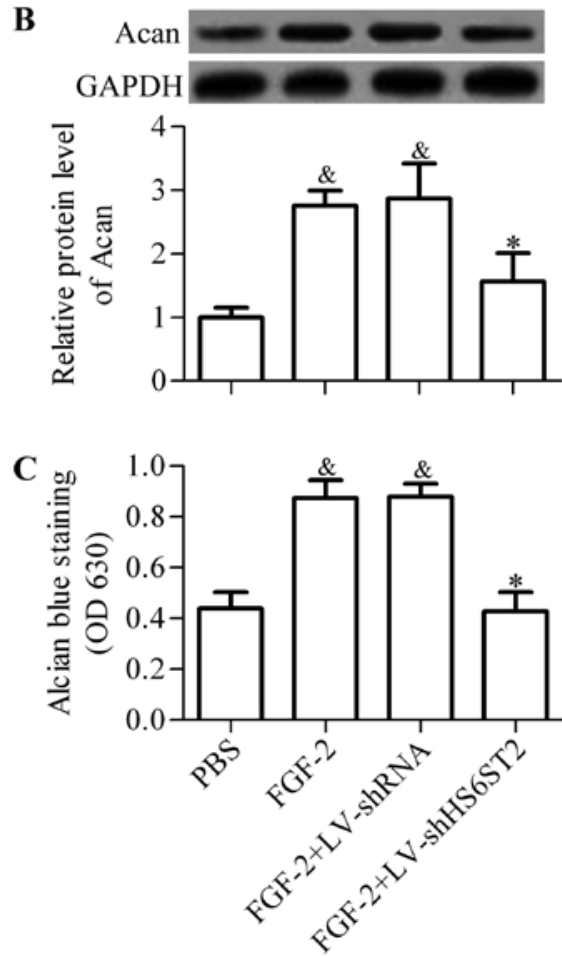

Figure 4. Effect of heparan sulfate 6-O-sulfotransferase-2 (HS6ST2) knockdown on fibroblast growth factor (FGF)-2-mediated chondrocyte differentiation. (A) RT-qPCR of the aggrecan (Acan) mRNA expression levels in the different cell groups. Cells were infected with lentiviral (LV)-shHS6ST2 overexpression vectors in the presence of FGF-2 $(5 \mathrm{ng} / \mathrm{ml})$ for 10 days $\mathrm{n}=3$; ${ }^{\& \&} \mathrm{p}<0.01$ vs. PBS group; ${ }^{* *} \mathrm{p}<0.01$ vs. FGF-2 or FGF- $2+$ LV-shRNA. (B) Western blot analysis of Acan protein expression in the different groups. Protein levels were quantified using Image-Pro Plus 6.0 software and normalized to GAPDH. $\mathrm{n}=3$; ${ }^{\&} \mathrm{p}<0.05$ vs. PBS group; " $\mathrm{p}<0.05$ vs. FGF-2 or FGF-2 + LV-shRNA. (C) Amount of proteoglycans detected by Alcian blue staining in the cell lysates. $n=3 ;{ }^{*} p<0.05$ vs. PBS group; ${ }^{*} p<0.05$ vs. FGF- 2 or FGF-2 + LV-shRNA

We then analyzed the effects of the silencing of HS6ST2 on FGF-2-mediated chondrocyte differentiation. The results from RT-qPCR demonstrated that the mRNA expression level of Acan, which was increased by treatment with FGF-2 during differentiation, was abrogated by the silencing of HS6ST2 (Fig. 4A). The results of western blot analysis further confirmed that the knockdown of HS6ST2 significantly decreased the protein expression level of Acan (Fig. 4B). Moreover, the silencing of HS6ST2 significantly decreased the amount of proteoglycans in the extracellular matrix (Fig. 4C). In conclusion, these data indicated that the knockdown of HS6ST2 blocked the chondrocyte differentiation induced by FGF-2.

Overexpression or knockdown of HS6ST2 affects SOX9 expression and the Wnt signaling pathway. To further delineate the role of HS6ST2 in regulating FGF-2-mediated signaling, the effects of HS6ST on the downstream gene, SOX9, were examined. The results revealed that the overexpression of HS6ST2 significantly enhanced both the mRNA (Fig. 5A) and protein (Fig. 5C) expression levels of SOX9 in the FGF-2-treated cells, whereas the silencing of HS6ST2 had the opposite effect (Fig. 5B and D). Furthermore, Wnt signaling activity was also regulated by HS6ST2. HS6ST2 overexpression downregulated Wnt activity (Fig. 5E), and HS6ST2 knockdown increased Wnt activity (Fig. 5F). Taken together, these data indicate that the modulation of HS6ST2 expression is involved in regulating SOX9 expression and Wnt signaling activity.

\section{Discussion}

In the present study, we demonstrated that HS6ST2 is involved in the regulation of chondrocyte differentiation by modulating FGF-2 signaling. We found that the FGF-2-mediated chondrocyte differentiation was enhanced by HS6ST2 overexpression and inhibited by the knockdown of HS6ST2. Furthermore, the major transcription factor for regulating chondrocyte differentiation, SOX9, which is regulated by FGF-2 signaling (20), was also regulated by the modulation of HS6ST2 expression. These data suggest that the dysregulation of HS6ST2 is involved in regulating endochondral ossification and is associated with skeletal dysplasias.

Over the years, the role of HS6ST2 has been widely investigated. It has been noted previously that HS6ST2, as well as HS6ST1, is overexpressed in ovarian cancer cells (24). The inhibition of HS6ST2 expression has been shown to effectively suppress the proliferation, migration and invasion of pancreatic cancer cells (11). Pollari et al (25) demonstrated that HS6ST2 is an important gene for transforming growth factor- $\beta$-induced interleukin-11 production in a mouse model of breast cancer bone metastasis. Recently, HS6ST2 was found to be abundantly expressed in colorectal cancer, and the increased expression of HS6ST2 was found to be associated with poor survival, which is considered a cancer-related marker (26). Di Maro et al (27) demonstrated that HS6ST2 was overexpressed in thyroid carcinoma samples, and that the silencing of HS6ST2 impaired cell invasion and migration. Aside from cancer, HS6ST2 has also been reported to be involved in embryonic stem cell differentiation (28) and idiopathic pulmonary fibrosis (29). In our previous study, we demonstrated that both the mRNA and protein levels of HS6ST2 were significantly downregulated in the cartilage of patients with osteoarthritis or Kashin-Beck disease (23). In particular, the silencing of HS6ST2 reduced cell viability and altered the expression of cartilage-related genes. Consistently, in another previous study, HS6ST2 exhibited an increased expression during cartilage tumour progression (30). These data indicate that HS6ST2 is relevant to chondrocytes. As a whole, these findings suggest that HS6ST2 is associated with signal transduction pathways, and thus, in regulating cellular processes in chondrocytes.

Previous studies have suggested that FGF is the most potent mitogen for chondrocytes, and that the 6-O-sulfation of heparan sulfate is required for receptor dimerization for FGF signaling $(31,32)$. It has been noted previously that 

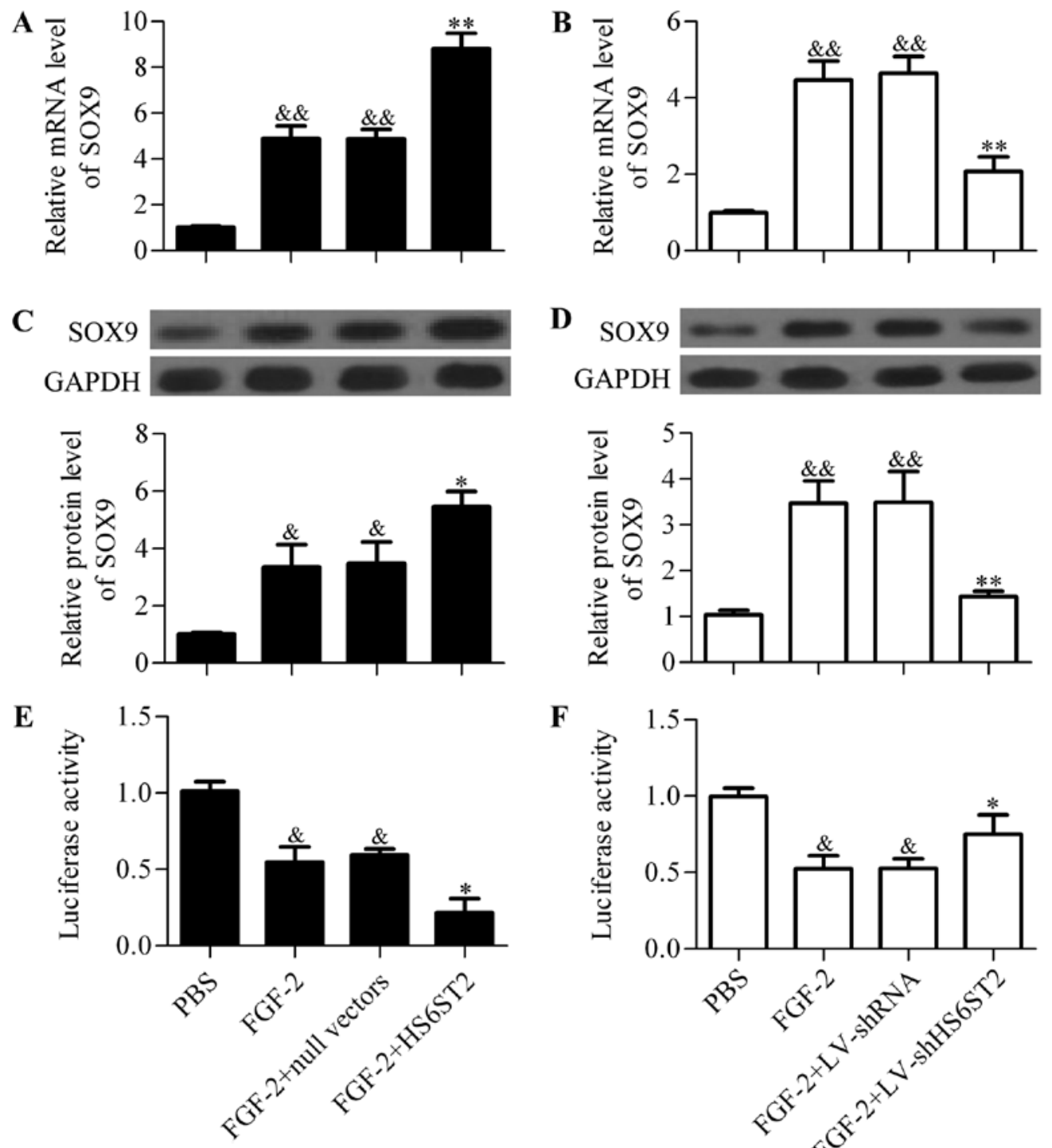

$\mathbf{F}$

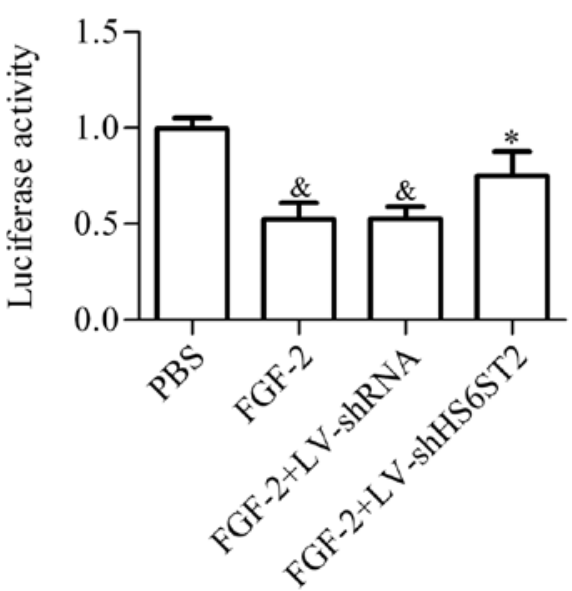

Figure 5. Modulation of heparan sulfate 6-O-sulfotransferase-2 (HS6ST2) expression is involved in regulating sex-determining region Y-type high mobility group box protein 9 (SOX9) expression and Wnt signaling activity. (A) RT-qPCR of SOX9 mRNA expression levels in pcDNA3.1-HS6ST2-=transfected cells. $\mathrm{n}=3$; \&\& $\mathrm{p}<0.01$ vs. PBS group; ${ }^{* *} \mathrm{p}<0.01$ vs. fibroblast growth factor (FGF)-2 or FGF-2 + null vectors. (B) RT-qPCR of SOX9 mRNA expression levels in lentiviral (LV)-shHS6ST2-infected cells. $n=3$; \&\& $<<0.01$ vs. PBS group; ${ }^{* *} \mathrm{p}<0.01$ vs. FGF-2 or FGF-2 + LV-shRNA. (C) Western blot analysis of the SOX9 protein expression level in pCDNA3.1-HS6ST2-transfected cells. Protein levels were quantified using Image-Pro Plus 6.0 and normalized to GAPDH. $n=3$; ${ }^{2}<<0.05$ vs. PBS group; , "p $<0.05$ vs. FGF-2 or FGF-2 + null vectors. (D) Western blot analysis of the SOX9 protein expression level in LV-shHS6ST2-infected cells. $\mathrm{n}=3$, \&\& $\mathrm{p}<0.01$ vs. PBS group; ${ }^{* *} \mathrm{p}<0.01$ vs. FGF-2 or FGF- $2+$ LV-shRNA. (E) Effect of HS6ST overexpression on Wnt signaling activity, as indicated by T-cell factor (TCF)-dependent TOPFlash reporter activity. After $24 \mathrm{~h}$ of transfection, the luciferase activity was examined. $\mathrm{n}=3 ;{ }^{*} \mathrm{p}<0.05 \mathrm{vs}$. PBSgroup; ${ }^{*} \mathrm{p}<0.05 \mathrm{vs}$. FGF-2 or FGF-2 + null vectors. (F) Effect of HS6ST2 knockdown on Wnt signaling activity, as indicated by TCF-dependent TOPFlash reporter activity. After $24 \mathrm{~h}$ of transfection, luciferase activity was examined. $\mathrm{n}=3$; ${ }^{\mathrm{k}} \mathrm{p}<0.05$ vs. PBS group; ${ }^{*} \mathrm{p}<0.05$ vs. FGF-2 or FGF- $2+\mathrm{LV}$-shRNA.

the inhibition of the expression of HST6ST2 significantly compromised FGF-2- and vascular endothelial growth factor (VEGF)-induced endothelial cell angiogenesis (33). It has also been reported that HS6ST2 plays a critical role in mediating energy metabolism by controlling signals from the FGF-19 subfamily proteins, and that HS6ST2 dysregulation is associated with glucose and insulin intolerance (34). The deletion of HS6ST1 and HS6ST2 has been shown to disrupt the FGF-10-FGFR2b-heparan sulfate complex on the cell surface, thus demonstrating the profound effect of FGF signaling on mammalian development (35). In the present study, we demonstrated that the FGF-2-mediated chondrocyte growth and differentiation was regulated by HS6ST2, that the overex- pression of HS6ST2 significantly enhanced FGF-2-mediated chondrocyte growth and differentiation, while the silencing of HSTST2 abrogated the FGF-2-mediated chondrocyte growth and differentiation. Therefore, our findings suggest that the HS6ST2-catalyzed 6-O-sulfation of heparan sulfate is essential for the FGF-2-mediated chondrocyte growth and differentiation.

Murakami et al (20) indicated that FGF-2 promotes chondrocyte differentiation through the induction of SOX9 and its downstream signaling, which is required for chondrocyte proliferation, differentiation and phenotypic stability $(22,36)$. It has previously been reported that SOX9 and Wnt/ $/$-catenin regulate chondrocyte proliferation and differentiation in a 
mutually antagonistic manner (37). Asai et al (38) reported that low-density lipoprotein receptor-related protein 4 accelerates chondrocyte differentiation by increasing SOX9 expression and inhibiting Wnt $/ \beta$-catenin activity. SOX9 is capable of facilitating the degradation of $\beta$-catenin, whereas $\beta$-catenin interacts with the C-terminal transactivation domain of SOX9, thereby blocking the function of SOX9 (37). In the present study, we found that HS6ST2 overexpression enhanced SOX9 expression and inhibited Wnt/ $\beta$-catenin activity in the FGF-2-treated chondrocytes, whereas the silencing of HS6ST2 caused the opposite effect. It is intriguing that unlike FGF and VEGF growth factors, the 6-O-sulphation of heparan sulfate inhibits the binding of Wnt to their receptors (39). Therefore, it is possible that HS6ST2 inhibits Wnt signaling activity, which plays a role in maintaining the function of SOX9 during chondrocyte differentiation.

In conclusion, the present study provides evidence that HS6ST2 is associated with chondrocyte growth and differentiation by modulating FGF-2 signaling. Our results demonstrated that 6-O-sulphation mediated by HS6ST2 played an important role in endochondral ossification. Therefore, it can be suggested that HS6ST2 is a potential and valuable molecular target for the treatment of skeletal dysplasias, such as dwarfism. However, this study only investigated the role of HS6ST2 in chondrocyte growth and differentiation in vitro. Therefore, further studies are warranted using animal models in vivo in order to delineate the precise mechanisms of action of HS6ST and its role in the regulation of chondrocyte growth and differentiation.

\section{Acknowledgements}

This study was supported by grants from the National Natural Science Foundation of China (nos. 81201426, 81201373 and $81301151)$ and the Key Project for Social Development and S\&T of Shaanxi (no. 2013K12-01-14).

\section{References}

1. Lefebvre V and Bhattaram P: Vertebrate skeletogenesis. Curr Top Dev Biol 90: 291-317, 2010.

2. Zhao Q, Eberspaecher H, Lefebvre V and De Crombrugghe B: Parallel expression of Sox 9 and Col2a1 in cells undergoing chondrogenesis. Dev Dyn 209: 377-386, 1997.

3. Yoshida CA, Kawane T, Moriishi T, Purushothaman A, Miyazaki T, Komori H, Mori M, Qin X, Hashimoto A, Sugahara K, et al: Overexpression of Galnt 3 in chondrocytes resulted in dwarfism due to the increase of mucin-type O-glycans and reduction of glycosaminoglycans. J Biol Chem 289: 26584-26596, 2014.

4. Holmbeck K, Bianco P, Caterina J, Yamada S, Kromer M, Kuznetsov SA, Mankani M, Robey PG, Poole AR, Pidoux I, et al: MT1-MMP-deficient mice develop dwarfism, osteopenia, arthritis, and connective tissue disease due to inadequate collagen turnover. Cell 99: 81-92, 1999.

5. Watanabe H, Nakata K, Kimata K, Nakanishi I and Yamada Y: Dwarfism and age-associated spinal degeneration of heterozygote cmd mice defective in aggrecan. Proc Natl Acad Sci USA 94 6943-6947, 1997.

6. Bernfield M, Götte M, Park PW, Reizes O, Fitzgerald ML, Lincecum J and Zako M: Functions of cell surface heparan sulfate proteoglycans. Annu Rev Biochem 68: 729-777, 1999.

7. Esko JD and Lindahl U: Molecular diversity of heparan sulfate. J Clin Invest 108: 169-173, 2001.

8. Ai X, Do AT, Kusche-Gullberg M, Lindahl U, Lu K and Emerson CP Jr: Substrate specificity and domain functions of extracellular heparan sulfate 6-O-endosulfatases, QSulf1 and QSulf2. J Biol Chem 281: 4969-4976, 2006.
9. Pye DA, Vives RR, Turnbull JE, Hyde P and Gallagher JT: Heparan sulfate oligosaccharides require 6-O-sulfation for promotion of basic fibroblast growth factor mitogenic activity. J Biol Chem 273: 22936-22942, 1998.

10. Sedita J, Izvolsky K and Cardoso WV: Differential expression of heparan sulfate 6-O-sulfotransferase isoforms in the mouse embryo suggests distinctive roles during organogenesis. Dev Dyn 231: 782-794, 2004.

11. Song K, Li Q, Peng YB, Li J, Ding K, Chen LJ, Shao CH, Zhang LJ and Li P: Silencing of hHS6ST2 inhibits progression of pancreatic cancer through inhibition of Notch signalling. Biochem J 436: 271-282, 2011.

12. Ashikari-Hada S, Habuchi H, Kariya Y, Itoh N, Reddi AH and Kimata K: Characterization of growth factor-binding structures in heparin/heparan sulfate using an octasaccharide library. J Biol Chem 279: 12346-12354, 2004.

13. Robinson CJ, Harmer NJ, Goodger SJ, Blundell TL and Gallagher JT: Cooperative dimerization of fibroblast growth factor 1 (FGF1) upon a single heparin saccharide may drive the formation of 2:2:1 FGF1.FGFR2c.heparin ternary complexes. J Biol Chem 280: 42274-42282, 2005.

14. Savage MP and Fallon JF: FGF-2 mRNA and its antisense message are expressed in a developmentally specific manner in the chick limb bud and mesonephros. Dev Dyn 202: 343-353, 1995.

15. Niswander L and Martin GR: Fgf-4 expression during gastrulation, myogenesis, limb and tooth development in the mouse. Development 114: 755-768, 1992.

16. Crossley PH and Martin GR: The mouse Fgf8 gene encodes a family of polypeptides and is expressed in regions that direct outgrowth and patterning in the developing embryo. Development 121: 439-451, 1995.

17. Gonzalez AM, Buscaglia M, Ong M and Baird A: Distribution of basic fibroblast growth factor in the 18-day rat fetus: localization in the basement membranes of diverse tissues. J Cell Biol 110: $753-765,1990$.

18. Jingushi S, Scully SP, Joyce ME, Sugioka Y and Bolander ME: Transforming growth factor-beta 1 and fibroblast growth factors in rat growth plate. J Orthop Res 13: 761-768, 1995.

19. Larbuisson A, Dalcq J, Martial JA and Muller M: Fgf receptors Fgfrla and Fgfr2 control the function of pharyngeal endoderm in late cranial cartilage development. Differentiation 86: 192-206, 2013.

20. Murakami S, Kan M, McKeehan WL and de Crombrugghe B: Up-regulation of the chondrogenic Sox9 gene by fibroblast growth factors is mediated by the mitogen-activated protein kinase pathway. Proc Natl Acad Sci USA 97: 1113-1118, 2000.

21. Akiyama H, Chaboissier MC, Martin JF, Schedl A and de Crombrugghe B: The transcription factor Sox 9 has essential roles in successive steps of the chondrocyte differentiation pathway and is required for expression of Sox 5 and Sox6. Genes Dev 16: 2813-2828, 2002.

22. Bi W, Deng JM, Zhang Z, Behringer RR and de Crombrugghe B: Sox 9 is required for cartilage formation. Nat Genet 22: 85-89, 1999.

23. Wang W, Zhong B, Sun J, Cao J, Tian J, Zhong N, Zhao W, Tian L, Xu P, Guo D, et al: Down-regulated HS6ST2 in osteoarthritis and Kashin-Beck disease inhibits cell viability and influences expression of the genes relevant to aggrecan metabolism of human chondrocytes. Rheumatology (Oxford) 50: 2176-2186, 2011.

24. Backen AC, Cole CL, Lau SC, Clamp AR, McVey R, Gallagher JT and Jayson GC: Heparan sulphate synthetic and editing enzymes in ovarian cancer. Br J Cancer 96: 1544-1548, 2007.

25. Pollari S, Käkönen RS, Mohammad KS, Rissanen JP, Halleen JM, Wärri A, Nissinen L, Pihlavisto M, Marjamäki A, Perälä M, et al: Heparin-like polysaccharides reduce osteolytic bone destruction and tumor growth in a mouse model of breast cancer bone metastasis. Mol Cancer Res 10: 597-604, 2012.

26. Hatabe S, Kimura H, Arao T, Kato H, Hayashi H, Nagai T, Matsumoto K, DE Velasco M, Fujita Y, Yamanouchi G, et al: Overexpression of heparan sulfate 6-O-sulfotransferase-2 in colorectal cancer. Mol Clin Oncol 1: 845-850, 2013.

27. Di Maro G, Orlandella FM, Bencivenga TC, Salerno P, Ugolini C, Basolo F, Maestro R and Salvatore G: Identification of targets of Twistl transcription factor in thyroid cancer cells. J Clin Endocrinol Metab 99: E1617-E1626, 2014

28. Gasimli L, Hickey AM, Yang B, Li G, dela Rosa M, Nairn AV, Kulik MJ, Dordick JS, Moremen KW, Dalton S and Linhardt RJ: Changes in glycosaminoglycan structure on differentiation of human embryonic stem cells towards mesoderm and endoderm lineages. Biochim Biophys Acta 1840: 1993-2003, 2014. 
29. Lu J, Auduong L, White ES and Yue X: Up-regulation of heparan sulfate 6-O-sulfation in idiopathic pulmonary fibrosis. Am J Respir Cell Mol Biol 50: 106-114, 2014.

30. Waaijer CJ, de Andrea CE, Hamilton A, van Oosterwijk JG, Stringer SE and Bovée JV: Cartilage tumour progression is characterized by an increased expression of heparan sulphate 6O-sulphation-modifying enzymes. Virchows Arch 461: 475-481, 2012.

31. Kato Y and Gospodarowicz D: Sulfated proteoglycan synthesis by confluent cultures of rabbit costal chondrocytes grown in the presence of fibroblast growth factor. J Cell Biol 100: 477-485, 1985.

32. Ornitz DM: FGFs, heparan sulfate and FGFRs: complex interactions essential for development. BioEssays 22: 108-112, 2000.

33. Ferreras C, Rushton G, Cole CL, Babur M, Telfer BA, van Kuppevelt TH, Gardiner JM, Williams KJ, Jayson GC and Avizienyte E: Endothelial heparan sulfate 6-O-sulfation levels regulate angiogenic responses of endothelial cells to fibroblast growth factor 2 and vascular endothelial growth factor. J Biol Chem 287: 36132-36146, 2012.

34. Nagai N, Habuchi H, Sugaya N, Nakamura M, Imamura T, Watanabe $\mathrm{H}$ and Kimata $\mathrm{K}$ : Involvement of heparan sulfate 6-O-sulfation in the regulation of energy metabolism and the alteration of thyroid hormone levels in male mice. Glycobiology 23: 980-992, 2013.
35. Qu X, Carbe C, Tao C, Powers A, Lawrence R, van Kuppevelt TH, Cardoso WV, Grobe K, Esko JD and Zhang X: Lacrimal gland development and Fgf10-Fgfr2b signaling are controlled by 2-Oand 6-O-sulfated heparan sulfate. J Biol Chem 286: 14435-14444, 2011.

36. Lefebvre $\mathrm{V}$ and de Crombrugghe $\mathrm{B}$ : Toward understanding SOX9 function in chondrocyte differentiation. Matrix Biol 16: 529-540, 1998

37. Akiyama H, Lyons JP, Mori-Akiyama Y, Yang X, Zhang R, Zhang Z, Deng JM, Taketo MM, Nakamura T, Behringer RR, et al: Interactions between Sox9 and beta-catenin control chondrocyte differentiation. Genes Dev 18: 1072-1087, 2004.

38. Asai N, Ohkawara B, Ito M, Masuda A, Ishiguro N and Ohno K: LRP4 induces extracellular matrix productions and facilitates chondrocyte differentiation. Biochem Biophys Res Commun 451: 302-307, 2014.

39. Lamanna WC, Kalus I, Padva M, Baldwin RJ, Merry CL and Dierks T: The heparanome - the enigma of encoding and decoding heparan sulfate sulfation. J Biotechnol 129: 290-307, 2007. 